Nouveaux cahiers de la recherche en éducation

\title{
Évolution des processus d'intégration sociale des élèves en fonction du profil de santé mentale au début du primaire
}

\section{Marcel Trudel, Cynthia Blais et Raphaëlle Marceau}

Volume 8, numéro 2, 2005

Groupe de recherche sur les inadaptations sociales de l'enfance

URI : https://id.erudit.org/iderudit/1017524ar

DOI : https://doi.org/10.7202/1017524ar

Aller au sommaire du numéro

Éditeur(s)

Faculté d'éducation, Université de Sherbrooke

ISSN

1911-8805 (numérique)

Découvrir la revue

Citer cet article

Trudel, M., Blais, C. \& Marceau, R. (2005). Évolution des processus d'intégration sociale des élèves en fonction du profil de santé mentale au début du primaire. Nouveaux cahiers de la recherche en éducation, 8(2), 7-16.

https://doi.org/10.7202/1017524ar
Résumé de l'article

L'objectif de la recherche est de mettre en évidence, auprès d'un échantillon d'élèves à risque, l'évolution longitudinale des profils d'intégration sociale en lien avec les variations des mesures de santé mentale relevées au début de la scolarisation. L'étude aborde également l'analyse des trajectoires sociométriques des filles et des garçons en lien avec les indices de santé mentale. Quatre évaluations ont été effectuées lors des deux premières années du primaire. Les résultats mettent en évidence que les garçons manifestant un trouble des conduites et un trouble d'opposition au début de la scolarisation sont rejetés et évalués négativement par les pairs au cours des deux premières années de scolarisation. Toutefois, chez les filles, on ne retrouve pas de trajectoires aussi déterminantes liant la santé mentale et l’intégration sociale.
Tous droits réservés @ Faculté d'éducation, Université de Sherbrooke,
Ce document est protégé par la loi sur le droit d'auteur. L'utilisation des services d’Érudit (y compris la reproduction) est assujettie à sa politique d'utilisation que vous pouvez consulter en ligne. 


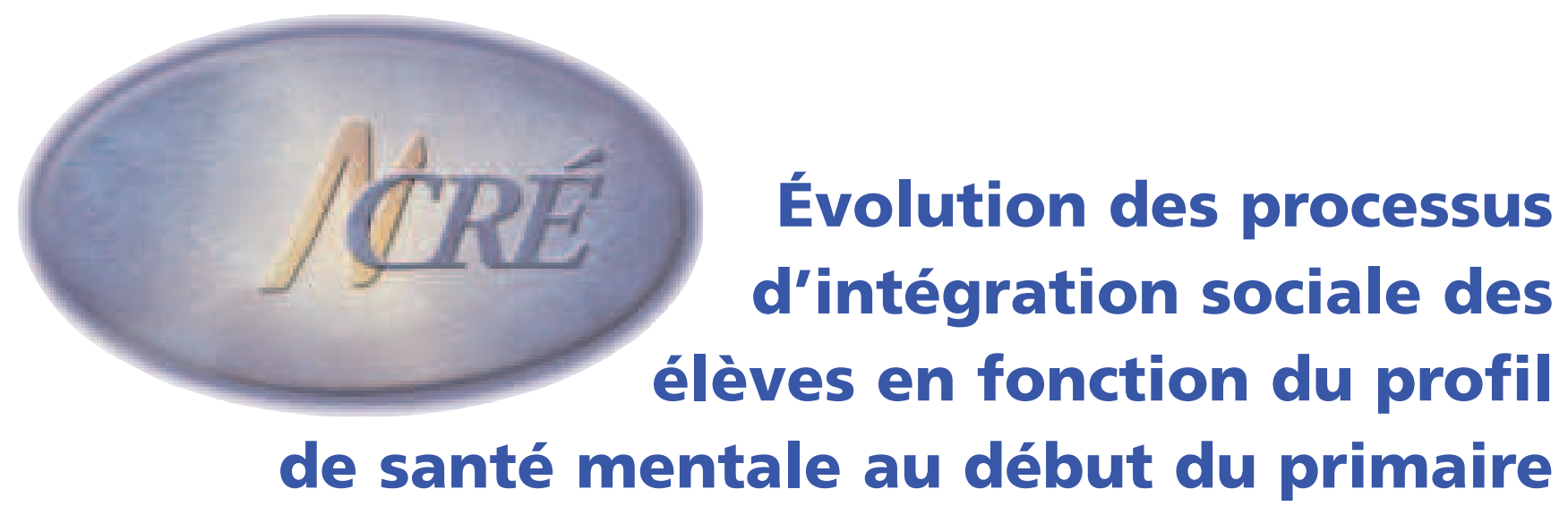

\author{
Marcel Trudel, Cynthia Blais \\ et Raphaëlle Marceau \\ Université de Sherbrooke
}

Résumé - L'objectif de la recherche est de mettre en évidence, auprès d'un échantillon d'élèves à risque, l'évolution longitudinale des profils d'intégration sociale en lien avec les variations des mesures de santé mentale relevées au début de la scolarisation. L'étude aborde également l'analyse des trajectoires sociométriques des filles et des garçons en lien avec les indices de santé mentale. Quatre évaluations ont été effectuées lors des deux premières années du primaire. Les résultats mettent en évidence que les garçons manifestant un trouble des conduites et un trouble d'opposition au début de la scolarisation sont rejetés et évalués négativement par les pairs au cours des deux premières années de scolarisation. Toutefois, chez les filles, on ne retrouve pas de trajectoires aussi déterminantes liant la santé mentale et l'intégration sociale.

\begin{abstract}
The goal of this study was to show the longitudinal evolution of social-integration profiles for a sample of at-risk pupils, as linked to variations in mental-health measures obtained at the start of schooling. The study also attempted to analyse girls' and boys' respective sociometric trajectories in relation to mental-health indices. Four assessments were carried out during the first two years of elementary school. Our findings show that boys who manifest behavioural and oppositional disorders at the start of their schooling are rejected and negatively evaluated by peers during their first two years of school. Trajectories linking mental health and social integration to the same degree of decisiveness are not observed for girls.
\end{abstract}




\section{Problématique}

L'intérêt de la présente démarche de recherche ${ }^{1}$ réside dans le fait que peu de travaux ont abordé le problème de la continuité et de la discontinuité sur l'aspect des préférences sociales (sur le plan sociométrique) des élèves en regard de leur profil de santé mentale en début de la scolarisation. On présume que la vie sociale en groupe de pairs pourrait représenter un soutien social et affectif déterminant dans le développement des élèves au cours des premières années du primaire. De plus, étant donné que de nombreux travaux concluent à une forte discrimination sociale basée sur le sexe, il est impératif de s'interroger sur la contribution de cette variable à la mise en place et à l'évolution des trajectoires de développement des élèves. De surcroît, on relève constamment dans les écrits que les filles et les garçons se distinguent au plan des indices associés aux troubles intériorisés et extériorisés (Dugré, Trudel et Valla, 2001). L'objectif prioritaire de la présente recherche consiste précisément à mettre en évidence l'évolution des profils sociométriques des filles et des garçons au cours des deux premières années du primaire en regard des variations des indices de santé mentale relevées en début de la scolarisation.

\subsection{Rôle des pairs sur le développement individuel des élèves}

L'importance du rôle des pairs sur le développement des enfants au cours de la période scolaire est indéniable. Ainsi, l'acquisition de la compétence sociale de l'élève serait directement tributaire des habiletés de l'enfant à s'ajuster aux différentes situations qui se présentent dans l'écologie sociale des pairs. Toutefois, il y a lieu de souligner que l'influence du groupe social n'est pas uniforme à travers tous les contextes de développement. Ainsi, selon Cillessen et Bukowski (2000), l'harmonie des relations entre les élèves est le reflet de l'équilibre complexe entre les forces cohésives (attraction sociale) et dispersives (rejet et agression) dans le groupe. L'organisation sociale du groupe imposerait des contraintes constantes sur le fonctionnement individuel des élèves au cours de leur développement (Strayer et Trudel, 1984). Nonobstant cette référence à l'écologie sociale, plusieurs chercheurs rapportent que la disponibilité d'un réseau de soutien social provenant du groupe de pairs est susceptible de représenter un puissant facteur de protection sur le développement socio-émotionnel de l'enfant (Booth, Rubin et Rose-Krasnor, 1998). En contrepartie, les élèves des classes du primaire qui sont rejetés d'une façon récurrente se révèlent, en comparaison à ceux qui sont socialement plus populaires, plus à risque de développer ultérieurement des troubles intériorisés et extériorisés, tout autant que des difficultés d'ordre scolaire et de décrochage scolaire (Coie, Terry, Lenox, Lochman et Hyman, 1995). D'ailleurs, ces élèves sont fréquemment la cible des interventions des professionnels du milieu scolaire.

Dans l'ensemble, on relève un nombre impressionnant de travaux ayant rapporté les effets négatifs associés au rejet des pairs sur le fonctionnement comportemental et le décrochage scolaire des élèves (Parker et Asher, 1987). Lorsque confrontés au rejet des pairs, ceux-ci sont dans une certaine mesure exposés à l'hostilité des autres et vivent quotidiennement du stress. Les

1 Les auteurs expriment leur reconnaissance aux enseignantes, à la direction de l'école et aux intervenants du CLSC qui ont collaboré à l'étude. Des remerciements particuliers s'adressent à tous les élèves qui ont accepté si généreusement de participer à cette recherche. Les auteurs ont apprécié la contribution de plusieurs étudiantes et étudiants de 2e et 3e cycles de la Faculté d'éducation de l'Université de Sherbrooke qui ont souvent travaillé bénévolement aux diverses phases de la collecte des données. 
résultats des études longitudinales démontrent que les élèves agressifs auraient également un profil à risque d'inadaptation à plus long terme (Farrington, 1991). La recension de Kupersmidt, Coie et Dodge (1990) met en évidence que l'exclusion des pairs est associée à l'apparition ultérieure de problèmes d'adaptation et en particulier à des troubles extériorisés. Toutefois, certains auteurs ont proposé d'établir une distinction entre les élèves qui seraient rejetés en raison de l'agressivité manifestée et ceux pour qui le rejet n'est pas associé à un profil antisocial (French, 1988). Ces deux styles d'élèves dits rejetés sont susceptibles de développer ultérieurement des problèmes d'adaptation de nature très distincte. Sur le plan typologique, on relève dans l'ensemble que la persistance du rejet social des pairs est susceptible d'occasionner progressivement une combinaison de trouble des conduites et d'hyperactivité (McArdle, O'Brien, Macmillan et Kolvin, 2000). De fait, le profil antisocial apparaîtrait comme le précurseur du rejet par les pairs qui déterminerait ultérieurement l'affiliation à des élèves déviants; en somme, on fait notamment l'hypothèse que l'émergence de problèmes extériorisés serait le reflet du cumul de cet effet séquentiel (Laird, Pettit, Dodge et Bates, 1999). White, Moffitt, Caspi, Jeglum-Bartusch, Needles et Stouthamer-Loeber (1994) rapportent que le profil agressif de l'élève lors des premières années de scolarisation prédit le développement de comportements antisociaux au cours de l'adolescence dans la mesure où celui-ci est associé à l'hyperactivité et à des problèmes d'inattention. Les travaux de Miller-Johnson, Coie, Maumary-Gremaud et Bierman (2002) fournissent une autre interprétation sur cette question. La forte corrélation entre le rejet des pairs et l'agressivité amène les auteurs à suggérer une contribution indépendante de l'exclusion sociale à l'émergence de trouble des conduites plutôt qu'à un effet attribuable uniquement au développement de l'agressivité. Cette conceptualisation du problème suggère d'étudier longitudinalement l'effet de l'exclusion sociale en contrôlant les variations du facteur agressivité et vice-versa. La combinaison du rejet social et de l'agressivité au cours de la période de l'enfance augmenterait à plus long terme les risques de développer des problèmes graves de comportements antisociaux.

L'analyse des trajectoires sociométriques des élèves ne se limite cependant pas à l'examen du lien entre le rejet social et l'agressivité puisque plusieurs travaux ont également investigué l'impact de l'isolement social sur le développement des problèmes de type intériorisé (Asendorpf, 1990 ; Cole, Martin et Peeke, 1999). L'ensemble de ces résultats, quoique moins nombreux que ceux en lien avec les problèmes extériorisés, met en évidence la pertinence de considérer l'influence conjointe des contraintes liées à l'adversité issue des rapports interpersonnels et de la vulnérabilité individuelle. Sur le plan théorique, il semble donc opportun de préconiser un modèle explicatif du développement qui accentue l'analyse de l'évolution des transactions complexes entre l'individu et son environnement social. L'isolement social et la victimisation, conséquences de l'exclusion par les pairs, pourraient engendrer à plus long terme un risque de manifestation de problèmes de type intériorisé comme de l'anxiété et de la dépression (Renouf, Kovacs et Mukerji, 1997; Gazelle et Ladd, 2003).

\subsection{Problèmes d'adaptation des filles et des garçons en groupe de pairs}

Précisons, d'entrée de jeu, que les travaux de recherche ayant abordé la problématique d'un trouble des conduites se sont principalement adressés à des échantillons de garçons. Les recherches qui ont utilisé un devis longitudinal ont surtout fait ressortir que, rétrospectivement, ces garçons avaient manifesté de sérieux problèmes de comportements à l'école primaire. Ainsi, Patterson, DeBaryshe et Ramsey (1989) rapportent que les garçons qui ont manifesté un haut taux d'agressivité et une conduite dysfonctionnelle lors de leur insertion à l'école primaire sont 
plus à risque de développer des comportements antisociaux au cours de la période de l'adolescence. Dans l'ensemble, les travaux de recherche démontrent que les garçons, comparativement aux filles, se comporteraient d'une façon plus agressive (Phillipsen, Bridges, McLemore et Saponaro, 1999); ces dernières se révélant plus prosociales et plus sensibles au point de vue des autres. Les différences entre les filles et les garçons sont plus importantes sur l'aspect des formes physiques d'agression et moins marquées au plan des comportements perturbateurs comme ceux associés au trouble d'opposition (Crick, 1997). Malgré le peu d'études longitudinales ayant incorporé des échantillons de filles, les résultats de ces travaux concluent que l'agressivité de ces dernières prédit ultérieurement, comme chez les garçons, un trouble des conduites (Broidy, Nagin, Tremblay, Bates, Brame, Dodge, Fergusson, Horwood, Loeber, Laird, Lynam, Moffitt, Pettit et Vitaro, 2003). Les troubles précoces d'opposition chez les filles représentent également, autant que l'agression physique, un bon prédicteur de trouble des conduites à l'adolescence. Il est intéressant de souligner que les formes moins physiques des comportements d'agression tendent à augmenter chez les filles et à diminuer chez les garçons au cours des premières années de scolarisation (Pulkkinen et Pitkanen, 1993).

Les précurseurs de la comorbidité sont également discutés par plusieurs chercheurs. Ainsi, chez les garçons, la présence de troubles extériorisés précoces augmente le risque de trouble des conduites et de troubles intériorisés à plus long terme alors que chez les filles, la précocité des deux types de troubles représenterait les seuls antécédents de problèmes intériorisés à l'âge adulte (Quinton, Rutter et Gulliver, 1990). Coie, Lochman, Terry et Hyman (1992) mettent également en évidence l'émergence d'un effet particulier chez les filles. En l'occurrence, il souligne que les problèmes extériorisés rapportés dans l'évaluation des parents sont davantage associés au rejet des pairs alors que dans l'évaluation auto-rapportée par leur fille c'est l'agressivité qui apparaît la cause première de l'inadaptation. En contrepartie, chez les garçons, c'est la coexistence du rejet des pairs et de l'agressivité qui prédirait la manifestation de troubles extériorisés à l'adolescence.

Considérant la complexité et la diversité des processus de socialisation liés à l'expérience de l'agressivité et de rejet par les pairs, il devient de plus en plus évident que l'étude des trajectoires de développement des enfants ne peut être abordée d'une façon linéaire ou selon un modèle causal unidirectionnel. Il y a nécessité de traiter le problème selon une approche plus multidimensionnelle où tant les variations intra-individuelles que inter-individuelles sont à considérer.

\subsection{Objectifs de l'étude}

Ce projet de recherche offre l'occasion de vérifier en premier lieu si le profil de santé mentale des élèves en début de scolarisation est associé à leur intégration sociale mesurée par l'approche sociométrique au cours des deux premières années du primaire. L'étude a pour objectif en deuxième lieu de tester si des différences émergent entre les filles et les garçons aux plans de la santé mentale et de l'intégration sociale, et ce, tout en vérifiant si les relations entre les deux construits distinguent les deux sous-groupes. Enfin, nous voulons examiner si les trajectoires longitudinales des filles et des garçons sont distinctes sur le plan des différentes caractéristiques sociales au regard de l'état de santé mentale initiale. 


\section{Méthodologie}

\subsection{Participants}

L'échantillon original ciblé comprend 70 élèves, dont 33 filles et 37 garçons, fréquentant une école primaire. La santé mentale ainsi que les caractéristiques des élèves ont été évaluées longitudinalement sur une période de deux ans à raison de deux sessions d'évaluation, soit en décembre et en mai de chaque année scolaire. Les élèves participent à un programme de prévention qui vise la promotion du développement personnel, psychologique et social. L'intervention privilégie une approche orientée vers l'expression des émotions et des peurs chez les élèves ainsi que vers l'organisation d'activités d'expression artistique. Les activités se déroulent chaque semaine à raison d'une période de 90 minutes en situation de groupe en présence de deux intervenants du CLSC et de l'enseignante. On estime qu'au total, une centaine d'heures ont été consacrées à l'intervention sur les deux années du projet.

\subsection{Instruments de mesure et déroulement des évaluations}

Après avoir obtenu l'autorisation requise, les enfants sont évalués collectivement dans leur classe respective pour compléter le questionnaire sur la santé mentale. Celle-ci est évaluée à l'aide du questionnaire Dominique- $R$ (Valla, Bergeron, Bérubé, Gaudet et Saint-Georges, 1994). Cet instrument utilise des stimuli visuels du personnage principal, Dominique (garçon ou fille), confronté à une série de situations concrètes qui illustrent les différents symptômes émotionnels ou comportementaux contenus dans le DSM-III-R. L'élève doit indiquer s'il ressemble ou non à Dominique. Ce questionnaire permet d'évaluer les sept indices de santé mentale les plus fréquents chez les enfants de 6 à 11 ans : la phobie simple, l'angoisse de séparation, l'anxiété, la dépression, l'hyperactivité avec déficit d'attention, le trouble d'opposition et le trouble des conduites. C'est la version 6 du questionnaire original Dominique-R qui est utilisée dans la présente étude. Le questionnaire comporte 96 vignettes réparties dans deux cahiers. Les résultats des travaux de recherche indiquent que ce questionnaire a une bonne validité de construit et prédictive, et une stabilité temporelle satisfaisante à partir d'échantillons cliniques ou d'élèves de classes régulières (Trudel, Rascalon, Ouellette et Dugré, 2003).

La seconde mesure utilisée concerne l'évaluation de l'intégration sociale des élèves à l'aide de l'approche sociométrique basée sur la procédure de nomination des pairs. Chaque élève se réfère aux photographies des enfants de la classe pour compléter cette tâche qui a été réalisée lors d'entrevues individuelles. Chaque élève doit identifier trois pairs de la classe qui sont les plus typiques (et, dans un second temps, les plus atypiques) sur chacune des composantes suivantes: la préférence sociale et le rejet des pairs, l'agressivité, la turbulence en classe, la coopération et l'humeur. Afin de rendre compte de la pondération reliée au choix de chaque élève dans l'analyse des résultats, le premier choix reçoit un score de 3 , le deuxième de 2 et le troisième de 1. Cette procédure est utilisée fréquemment par les chercheurs désirant évaluer le statut sociométrique des membres du groupe et se révèle en général valide et stable (Newcomb et Bukowski, 1984). 


\section{Résultats}

Les résultats se rapportant aux échelles de symptômes du Dominique- $R$ sont d'abord transformés en proportions afin de rendre compte du nombre inégal d'items associés à chacune des sept catégories. Les proportions moyennes de symptômes aux deux premières évaluations sont par la suite agrégées afin d'assurer une meilleure représentation de la santé mentale des élèves au cours de la première année de scolarisation. Les résultats des analyses réalisées à l'aide de tests-t concernant les différences de moyennes entre les filles et les garçons à chacune des échelles du Dominique-R ne font ressortir aucun effet significatif. Les variations aux échelles sociométriques distinguent toutefois les filles et les garçons sur plusieurs indices (voir tableau 1). Soulignons en premier lieu que les effets se rapportant aux indices de la préférence sociale et du rejet des pairs sont plutôt atténués puisqu'on observe un seul écart significatif, soit celui où les garçons sont plus rejetés que les filles à l'évaluation de l'automne de la seconde année (T3). Quant aux moyennes associées aux caractéristiques sociales des élèves, celles-ci différencient nettement les filles et les garçons. On note que plusieurs des variables révèlent des différences de moyennes qui sont défavorables aux garçons, et ce, principalement aux catégories agressif, turbulent et non-coopération.

Tableau 1

Scores moyens aux indices sociométriques pour chacune des sessions d'évaluation (T1 à T4) en fonction du sexe des élèves

\begin{tabular}{|l|c|c|c|c|c|c|c|c|c|c|c|c|}
\hline Temps d'évaluation & \multicolumn{9}{|c|}{ T1 } & \multicolumn{9}{|c|}{ T2 } & \multicolumn{3}{|c|}{ T3 } \\
\hline Catégories sociales & $\mathrm{F}$ & $\mathrm{G}$ & $t$ & $\mathrm{~F}$ & $\mathrm{G}$ & $t$ & $\mathrm{~F}$ & $\mathrm{G}$ & $t$ & $\mathrm{~F}$ & $\mathrm{G}$ & $t$ \\
\hline Préférence/rejet social \\
\hline Populaire & 5,4 & 6,2 & $-0,7$ & 5,0 & 6,0 & $-0,7$ & 5,5 & 6,2 & $-0,6$ & 5,7 & 5,5 & 0,2 \\
\hline Rejeté & 4,4 & 6,9 & $-1,5$ & 4,0 & 6,8 & $-1,5$ & 4,3 & 7,4 & $-1,9 *$ & 4,6 & 5,6 & $-0,6$ \\
\hline Caractéristiques sociales \\
\hline Agressif & 2,7 & 9,2 & $-2,0 *$ & 3,0 & 8,4 & $-1,9 *$ & 2,9 & 9,6 & $-2,3 *$ & 2,9 & 9,1 & $-3,0 * *$ \\
\hline Non agressif & 7,3 & 5,2 & 1,3 & 7,1 & 5,0 & 1,6 & 6,9 & 5,2 & 1,3 & 8,5 & 3,7 & $2,6^{*}$ \\
\hline Turbulent & 2,7 & 9,8 & $-2,8 * *$ & 2,5 & 8,9 & $-3,0 * *$ & 3,1 & 9,3 & $-2,8 * *$ & 2,9 & 9,2 & $-3,1 * *$ \\
\hline Non turbulent & 7,3 & 5,0 & 1,5 & 7,6 & 4,3 & $2,4 *$ & 7,5 & 4,5 & $2,0 *$ & 7,8 & 4,4 & 1,8 \\
\hline Coopératif & 7,7 & 4,8 & 1,5 & 7,0 & 5,0 & 1,2 & 6,3 & 6,0 & 0,2 & 7,6 & 4,4 & $2,0 *$ \\
\hline Non coopérative & 4,0 & 8,5 & $-2,1 *$ & 3,8 & 7,6 & $-2,2 *$ & 4,1 & 8,1 & $-2,3 *$ & 4,4 & 7,2 & $-1,5$ \\
\hline Bonne humeur & 5,7 & 6,7 & 0,6 & 6,9 & 4,9 & 1,7 & 6,6 & 5,4 & 1,0 & 6,9 & 5,1 & 1,2 \\
\hline Mauvaise humeur & 4,8 & 7,4 & 1,1 & 4,0 & 7,4 & $-1,8$ & 5,0 & 7,3 & $-1,2$ & 4,0 & 7,8 & $-2,1 *$ \\
\hline
\end{tabular}

Légende: $\mathrm{F}=$ Filles; $\mathrm{G}=$ Garçons; $* \mathrm{p}<0,05 ; * * \mathrm{p}<0,01$.

La deuxième série d'analyses vise à vérifier les corrélations entre la santé mentale et les indices liés aux caractéristiques sociométriques. Les résultats ne font ressortir aucune relation significative entre les préférences sociales et les indices de santé mentale. C'est plutôt le rejet par les pairs qui se révèle constamment corrélé au trouble des conduites et ce, aux quatre sessions d'évaluation. Quant aux matrices de corrélation intégrant les différentes composantes des caractéristiques sociales et la santé mentale, celles-ci mettent en évidence 30 effets significatifs $(\mathrm{p}<0,05)$ dont $87 \%$ impliquent la catégorie trouble des conduites. Ces analyses ont été reprises, mais cette fois indépendamment pour les filles et les garçons. Dans l'ensemble, les 
co-variations apparaissent plus fréquentes chez les garçons puisque $70 \%$ des corrélations significatives se rapportent à ces derniers (26 effets sur 37 avec $p<0,5)$; la presque totalité de ces effets concerne le trouble des conduites (19 effets) et le trouble d'opposition (5 effets).

La dernière étape d'analyse a permis de mieux décrire les principaux effets relevés sur le plan de l'approche corrélationnelle. Ainsi, les scores des variables de la santé mentale étant les plus associés aux représentations sociales ont été standardisés en tenant compte des variations individuelles et de leur distribution. Deux sous-groupes par catégorie sont identifiés selon que les moyennes des symptômes soient élevées ou basses. Les mesures des variables dépendantes considérées seront d'abord réduites afin de faciliter l'interprétation des effets. Des analyses factorielles en composantes principales avec rotation varimax sont appliquées aux données sur les huit catégories des caractéristiques sociales, et ce, pour chacune des sessions d'évaluation. D'une façon systématique, deux facteurs sont extraits pour chacune des sessions avec des valeurs eigen supérieures à 1 . À noter que les variances expliquées à travers les quatre sessions augmentent progressivement passant de $69 \%$ au T1 à 81,6\% au T4. Les premiers facteurs regroupent les échelles associées aux représentations sociales négatives comme l'agressivité, la turbulence, la non-coopération et la mauvaise humeur alors que les saturations aux seconds facteurs incorporent les quatre variables décrivant les représentations positives des pairs. Par la suite, des analyses contrastant les différences de moyennes sur les variables sociales sont réalisées à l'aide de tests-t, et ce, indépendamment pour les filles et les garçons. La figure 1 fournit un sommaire des principaux effets pour chacune des échelles de la santé mentale se révélant les plus révélatrices en regard des scores factoriels issus des représentations sociales négatives et positives.

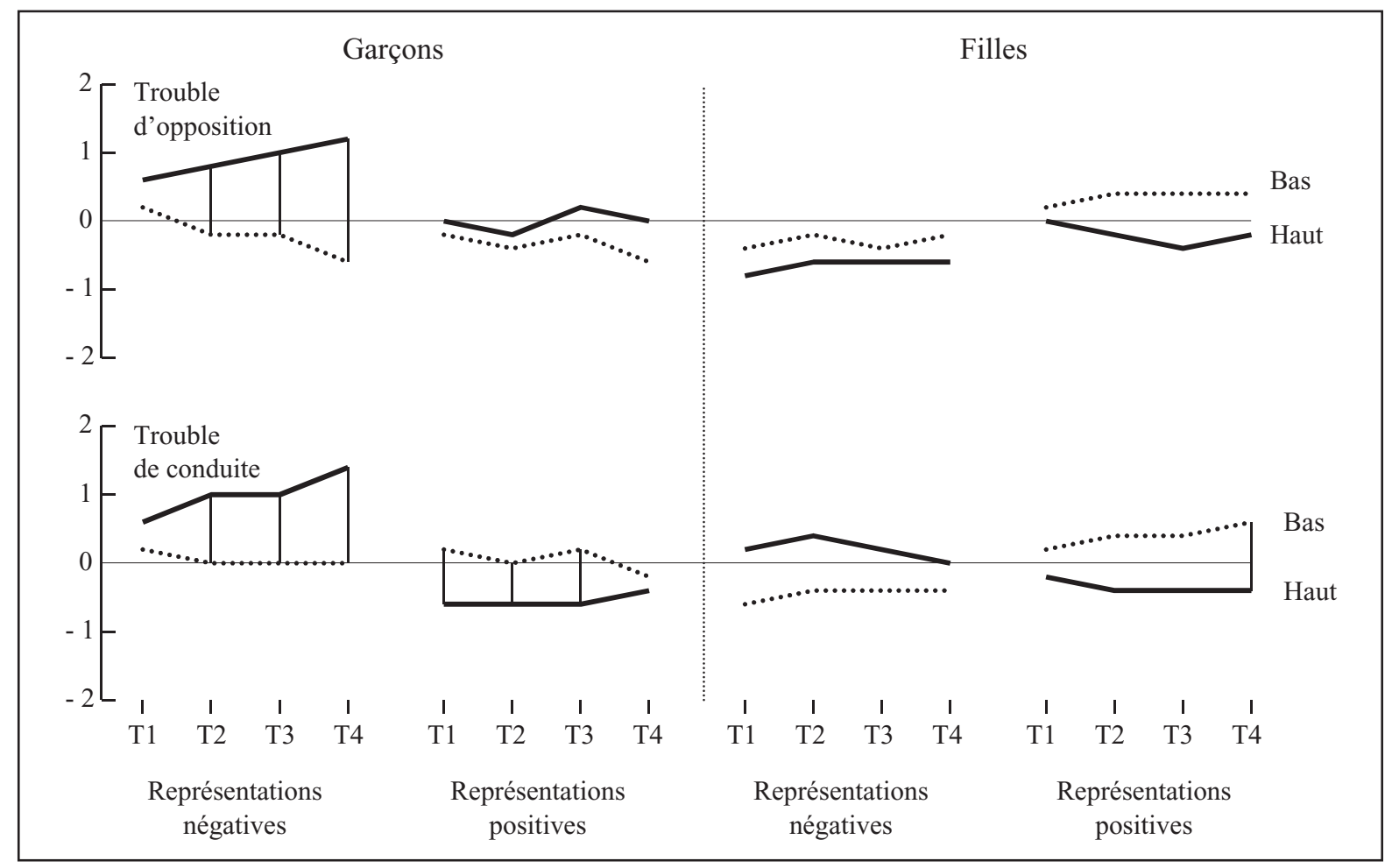

Figure 1 - Profils longitudinaux des garçons et des filles sur les indices sociométriques (scores factoriels) en fonction des résultats sur la santé mentale (troubles des conduites et d'opposition) 
Dans l'ensemble, ces résultats mettent en évidence, sur le plan des trajectoires longitudinales, que les représentations initiales des garçons se rapportant au trouble des conduites et au trouble d'opposition exacerbent progressivement les représentations négatives des pairs. Quant aux représentations positives des pairs, celles-ci sont liées au trouble des conduites lors des trois premières évaluations des garçons et à la fin de la seconde année chez les filles. En général, la trajectoire des garçons sur le plan social est beaucoup plus affectée par deux symptômes des troubles extériorisés (troubles des conduites et d'opposition). Enfin, le profil des filles apparaît moins relié à la représentation de leur santé mentale.

\section{Discussion et conclusion}

L'intérêt de la présente recherche était de cerner une relation possible entre les représentations initiales ( $1^{\text {re }}$ année $)$ de l'élève de son état de santé mentale et celles des pairs sur le plan du fonctionnement social au cours des deux premières années de scolarisation. Historiquement, le problème est pertinent, tant au plan fondamental qu'empirique, puisque le propos aborde le lien complexe entre le processus de construction de soi (en regard des auto-représentations) et de son impact sur les représentations d'autrui ou sur la réputation sociale au cours de l'enfance. En d'autres termes, est-ce que la représentation de l'élève de son comportement est validée par l'évaluation de l'entourage social des pairs d'une façon cohérente et constante? Il faut également souligner que cette recherche s'est déroulée lors de l'implantation d'un programme intensif de prévention psychosociale. Dans ce contexte, on doit s'attendre à ce que les variations au cours de la période d'intervention ne suivent pas nécessairement les tendances rapportées dans les écrits scientifiques en regard des effets du rejet par les pairs sur la santé mentale des élèves pour des échantillons populationnels et atypiques. De fait, on doit prévoir que l'intervention pourrait induire dans une certaine mesure des changements sur le fonctionnement social du groupe et sur les représentations des élèves. Toutefois, compte tenu de l'absence d'un groupe témoin, il s'avère impossible de conclure que l'intervention a produit les effets escomptés. Cette contrainte représente une limite importante de la présente étude.

Un des constats qui se dégage des résultats met en lumière l'émergence, chez les garçons, du lien entre le rejet social et les représentations du trouble des conduites. Cette association se généralise aux indices décrivant les différentes caractéristiques sociales des élèves. Toutefois, les effets se rapportent d'une façon constante aux aspects négatifs des représentations. Chez les filles, aucune convergence n'émerge de nos analyses en ce qui concerne la relation entre la santé mentale et l'intégration sociale. Les contrastes entre les sous-groupes définis en termes de profils extrêmes confirment que les troubles des conduites et d'opposition perçus par les garçons ont un impact persistant sur les représentations sociales des pairs au cours des deux années du projet. Ce résultat rejoint les conclusions de plusieurs chercheurs à l'effet que le rejet par les pairs au cours des premières années de scolarisation est associé à des troubles extériorisés (Kupersmidt et al., 1990). Nos résultats confirment que cet effet persiste au cours des deux premières années de scolarisation. Toutefois, la provenance des rejets sociaux n'a pas été abordée dans cette étude. Ainsi, le rejet des garçons par les pairs est-il issu des représentations des filles, des garçons ou de l'ensemble des élèves? Cette information permettrait probablement de mieux cerner le profil sociométrique des filles et le lien avec la santé mentale. L'absence de relation entre les problèmes intériorisés et l'état de santé mentale, tant chez les filles que les garçons, ne confirme pas les résultats des travaux de Cole et al. (1999). Un tel résultat pourrait être attribuable au fait que le suivi longitudinal ne s'est pas prolongé jusqu'à la fin de la période du primaire. 


\section{Références}

Abikoff, H. et Gittelman, R. (1985). The normalizing effects of methylphenidage on the classroom behavior of ADHD children. Journal of Abnormal Child Psychology, 13(1), 33-44.

Asendorpf, J.B. (1990). Beyond social withdrawal: Shyness, unsociability and peer avoidance. Human Development, 33(4-5), 250-259.

Booth, C.L., Rubin, K.H. et Rose-Krasnor, L. (1998). Perceptions of emotional support from mother and friend in middle childhood: Links with social-emotional adaptation and preschool attachment security. Child Development, 69(2), 427-443.

Broidy, L.M., Nagin, D.S., Tremblay, R.E., Bates, J.E., Brame, B., Dodge, K.A., Fergusson, D., Horwood, J.L., Loeber, R., Laird, R., Lynam, D.R., Moffit, T.E., Pettit, G.S. et Vitaro, F. (2003). Developmental trajectories of childhood disruptive behavior and adolescent delinquency: A six site, cross-national study. Developmental Psychology, 39(2), 222-245.

Cillessen, A.H.N. et Bukowski, W.M. (2000). Recent advances in the measurement of acceptance and rejection in the peer system. New Direction for Child and Adolescent Development, 88, 3-10.

Coie, J.D., Lochman, J.E., Terry, R. et Hyman, C. (1992). Predicting early adolescent disorder from childhood aggression and peer rejection. Journal of Consulting and Clinical Psychology, 60(5), 783-792.

Coie, J.D., Terry, R., Lenox, K., Lochman, J. et Hyman, C. (1995). Childhood peer rejection and aggression as predictors of stable patterns of adolescent disorder. Development and Psychopathology, 7(4), 697-713.

Cole, D.A., Martin, J.M. et Peeke, L.A. (1999). Children's over and underestimation of academic competence: A longitudinal study of gender differences, depression, and anxiety. Child Development, 70(2), 459-473.

Crick, N.R. (1997). Engagement in gender normative versus nonnormative forms of aggression: Links to socialpsychological adjustment. Developmental Psychology, 33(4), 610-617.

Dugré, S., Trudel, M. et Valla, J.P. (2001). Considérations individuelles et culturelles en santé mentale des enfants: le Dominique à l'épreuve. Revue canadienne de psychoéducation, 30(1), 119-138.

Farrington, D. (1991). Childhood aggression and adult violence: Early precursors and later life outcome. In D. Peppler et K. Rubin (dir.), The development and treatment of childhood aggression. Hillsdale, NJ : Lawrence Erlbaum.

French, D.C. (1988). Heterogeneity of peer rejected boys: Aggressive and nonaggressive subtypes. Child Development, 59(4), 976-985.

Gazelle, H. et Ladd, G.W. (2003). Anxious solitude and peer exclusion: A diathesis-stress model of internalizing trajectories in childhood. Child Development, 74(1), 257-278.

Kupersmidt, J., Coie, J. et Dodge, K.A. (1990). The role of peer relations in the development of disorders. In S. Asher et J. Coie (dir.), Peer rejection childhood, New York, NY: Cambridge University Press.

Laird, R.D., Pettit, G.S., Dodge, K.A. et Bates, J.E. (1999). Brest friendships, group relationships and antisocial behavior in early adolescence. Journal of Early Adolescence, 19(4), 413-437.

McArdle, P., O'Brien, G., Macmillan, A. et Kolvin, I. (2000). The peer relations of disruptive children with reference to hyperactivity and conduct disorder. European Child and Adolescent Psychiatry, 9(2), 91-99.

Miller-Johnson, S., Coie, J.D., Maumary-Gremaud, A. et Bierman, K. (2002). Peer rejection and aggression and early starter models of conduct disorder. Journal of Abnormal Child Psychology, 30(3), 217-230.

Newcomb, A.F. et Bukowski, W.M. (1984). A longitudinal study of the utility of social preference and social impact sociometric classification schemes. Child Development, 55(4), 1434-1447.

Parker, J.G. et Asher, S.R. (1987). Peer relations and later personal adjustment: Are low-accepted children «at risk»? Psychological Bulletin, 102(3), 357-389.

Patterson, DeBaryshe et Ramsey (AJOUTER INITIALES des auteurs)(1989). A developmental perspective on antisocial behavior. American Psychologist, 44(2), 329-335.

Phillipsen, L.C., Bridges, S.K., McLemore, T.G. et Saponaro, L.A. (1999). Perceptions of social behavior and peer acceptance in kindergarten. Journal of Research in Childhood Education, 14(1), 68-77. 
Pulkkinen, L. et Pitkanen, T. (1993). Continuities in aggressive behavior from childhood to adulthood. Aggressive Behavior, 19(4), 249-263.

Quinton, D., Rutter, M. et Gulliver, L. (1990). Continuities in psychiatric disorders from childhood to adulthood in the children of psychiatric patients. In L.N. Robins et M. Rutter (dir.), Straight and devious pathways from childhood to adulthood. New York, NY: Cambridge University Press.

Renouf, A.G., Kovacs, M. et Mukerji, P. (1997). Relationship of depressive, conduct, and comorbid disorders and social functioning in childhood. Journal of the American Academy of Child and Adolescent Psychiatry, 36(7), 998-1004.

Strayer, F.F. et Trudel, M. (1984). Developmental changes in the nature and function of social dominance among young children. Ethology and Sociobiology, 5(4), 279-295.

Trudel, M., Rascalon, E., Ouellette, R. et Dugré, S. (2003). Analyse longitudinale de l'effet d'un programme d'intervention précoce sur la santé mentale d'élèves provenant de milieu socioéconomiquement faible. In F. Larose (dir.), Difficultés d'adaptation sociale ou scolaire et intervention éducative (p. 111-137) Sherbrooke: Éditions du CRP.

Valla, J.P., Bergeron, L., Bérubé, H., Gaudet, N. et Saint-Georges, M. (1994). A structured pictorial questionnaire to assess DSM-III-R-based diagnoses in children (6-11 years) : Development, validity and reliability. Journal of Abnormal Child Psychology, 22(4), 403-423.

White, J., Moffitt, T.E., Caspi, A., Jeglum-Bartusch, D., Needles, D. et Stouthamer-Loeber, M. (1994). Measuring impulsivity and examining its relation to delinquency. Journal of Abnormal Psychology, 103(2), 192-205. 